\title{
Identification of bioactive metabolites in human iPSC-derived dopaminergic neurons with PARK2 mutation: Altered mitochondrial and energy metabolism
}

\author{
Justyna Okarmus, ${ }^{1}$ Jesper F. Havelund, ${ }^{2}$ Matias Ryding, ${ }^{1}$ Sissel I. Schmidt, ${ }^{1}$ Helle Bogetofte, ${ }^{1}$ \\ Rachel Heon-Roberts, ${ }^{3}$ Richard Wade-Martins, ${ }^{3}$ Sally A. Cowley, ${ }^{4}$ Brent J. Ryan, ${ }^{3}$ Nils J. Færgeman, ${ }^{2}$ \\ Poul Hyttel, 5 and Morten Meyer ${ }^{1,6,7, *}$ \\ ${ }^{1}$ Department of Neurobiology Research, Institute of Molecular Medicine, University of Southern Denmark, J.B. Winsløws Vej 21, 5000 Odense C, Denmark \\ ${ }^{2}$ Department of Biochemistry and Molecular Biology, VILLUM Center for Bioanalytical Sciences, University of Southern Denmark, Campusvej 55, 5230 \\ Odense M, Denmark \\ ${ }^{3}$ Oxford Parkinson's Disease Center, Department of Physiology, Anatomy and Genetics, University of Oxford, South Parks Road, Oxford OX1 3QX, UK \\ ${ }^{4}$ James Martin Stem Cell Facility, Sir William Dunn School of Pathology, University of Oxford, South Parks Road, Oxford OX1 3RE, UK \\ ${ }^{5}$ Department of Veterinary and Animal Sciences, Faculty of Health and Medical Sciences, University of Copenhagen, Grønnegaardsvej 7, 1870 Frederiksberg \\ C, Denmark \\ ${ }^{6}$ Department of Neurology, Odense University Hospital, J.B. Winsløws Vej 4, 5000 Odense C, Denmark \\ ${ }^{7}$ BRIDGE - Brain Research Inter-Disciplinary Guided Excellence, Department of Clinical Research, University of Southern Denmark, J.B. Winsløws Vej 19, \\ 5000 Odense C, Denmark \\ *Correspondence: mmeyer@health.sdu.dk \\ https://doi.org/10.1016/j.stemcr.2021.04.022
}

\section{SUMMARY}

PARK2 (parkin) mutations cause early-onset Parkinson's disease (PD). Parkin is an ubiquitin E3 ligase that participates in several cellular functions, including mitochondrial homeostasis. However, the specific metabolomic changes caused by parkin depletion remain unknown. Here, we used isogenic human induced pluripotent stem cells (iPSCs) with and without PARK2 knockout (KO) to investigate the effect of parkin loss of function by comparative metabolomics supplemented with ultrastructural and functional analyses. PARK2 KO neurons displayed increased tricarboxylic acid (TCA) cycle activity, perturbed mitochondrial ultrastructure, ATP depletion, and dysregulation of glycolysis and carnitine metabolism. These perturbations were combined with increased oxidative stress and a decreased anti-oxidative response. Key findings for PARK2 KO cells were confirmed using patient-specific iPSC-derived neurons. Overall, our data describe a unique metabolomic profile associated with parkin dysfunction and show that combining metabolomics with an iPSC-derived dopaminergic neuronal model of PD is a valuable approach to obtain novel insight into the disease pathogenesis.

\section{INTRODUCTION}

The pathological hallmark of Parkinson's disease (PD) is the marked loss of dopaminergic neurons in the substantia nigra pars compacta (SNc), causing dopamine depletion in the striatum (Caviness, 2014). Although the multifactorial etiology of PD and the pathological mechanisms underlying the neuronal degeneration remain largely undetermined, various PD-related genetic-environmental interactions are thought to contribute to the pathogenesis of the disease. Mitochondrial dysfunction associated with oxidative stress and energy failure is increasingly thought to be implicated in PD (Dias et al., 2013; Schapira, 2009).

Mutations in a number of genes have been found to cause monogenic forms of PD with both recessive (e.g., PARK2, PINK1, DJ-1) and autosomal dominant transmission (e.g., LRRK2, SNCA) (Bonifati et al., 2003; Lucking et al., 2000). Loss-of-function mutations of the PARK2 gene, encoding the cytosolic E3 ubiquitin protein ligase parkin, account for a large proportion of familial earlyonset PD cases. Even though monogenic (often familial) forms of PD are not frequent (less than 5\% of all PD cases), elucidation of their molecular mechanisms could help identify causes of the more common sporadic forms of the disease (Reed et al., 2019). The protein parkin is thought to play an essential role in the regulation of mitochondrial homeostasis and dynamics. Increased expression of the PARK2 gene transcript confers protection from stressinduced cell death (Dias et al., 2013). Furthermore, loss of parkin increases susceptibility to stress and death in primary cells (Anandhan et al., 2017; Schapira, 2009). It is thus believed that PARK2-genetic causes of PD likely involve a loss-of-function phenotype that leads to the clinical presentation of the disease (Lesage and Brice, 2009).

Mitochondria are the powerhouses of the cell and play an important role in various metabolic pathways. Apart from their crucial role in cellular energy metabolism (ATP production via oxidative phosphorylation [OXPHOS]), mitochondria are powerful organelles that regulate reactive oxygen species (ROS) production, $\mathrm{NAD}^{+} / \mathrm{NADH}$ ratio, and programmed cell death (Anandhan et al., 2017; Zorov et al., 2014). Disturbances in mitochondrial function have been suggested to contribute to neurodegeneration (Chan, 2020). Evidence of mitochondrial impairment in PD comes from mitochondrial toxin-induced models of the disease (Martinez and Greenamyre, 2012), and from the examination of mitochondria in postmortem tissue from patients with idiopathic PD (Parker et al., 2008; 
A

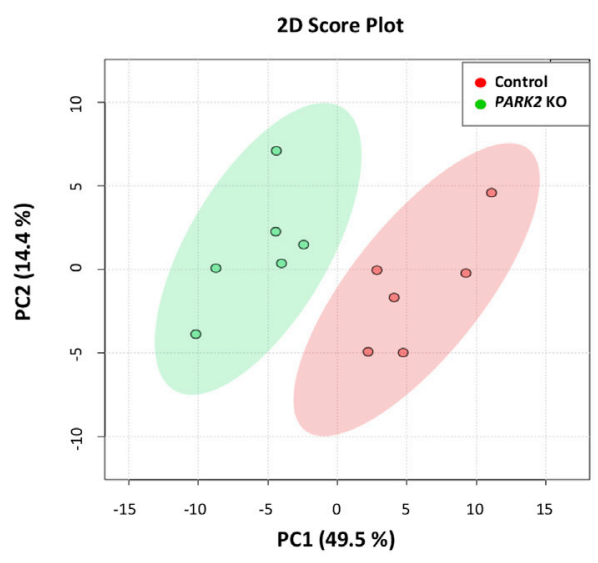

B

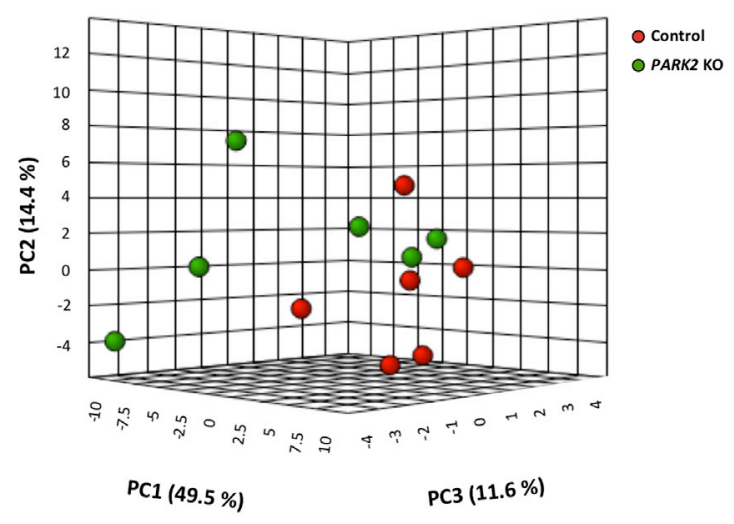

C
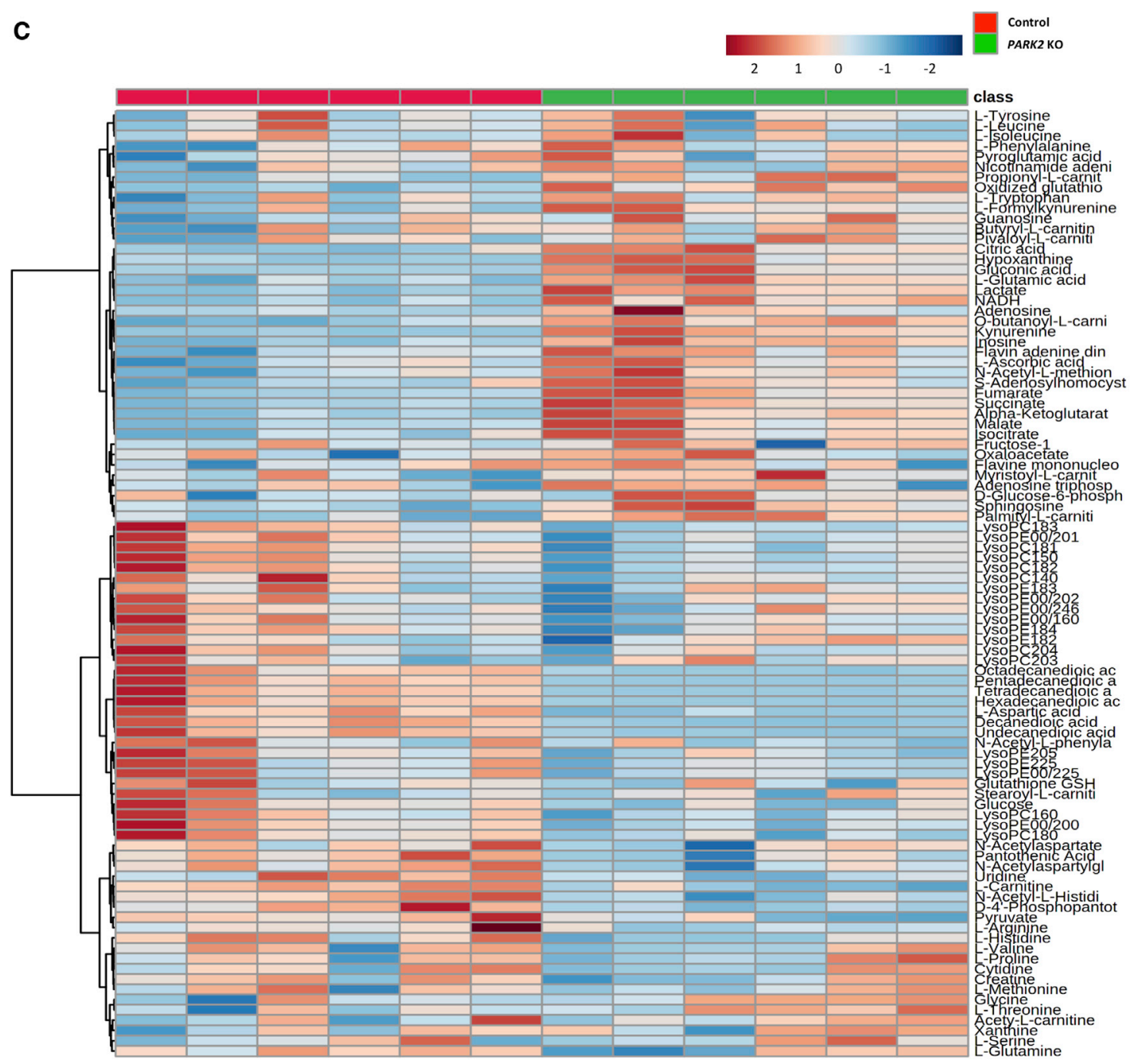

Figure 1. Comparative metabolic profiling of isogenic PARK2 KO and control neurons

(A and B) (A) 2D and (B) 3D PCA score plots showing a significant separation between PARK2 K0 neurons and control neurons (k-mean clustering algorithm, $95 \%$ confidence region). The observations are coded according to class membership: green, PARK2 K0 neurons; red, control neurons. 
Schapira, 2008). Disease-causing mutations in genes encoding for proteins with a mitochondrial function (PARK2, PINK1, DJ-1, HTRA2) have also been identified, further supporting the conclusion that mitochondrial dysfunction is a hallmark of PD (Bjorkblom et al., 2014; Fitzgerald et al., 2012; Pickrell and Youle, 2015). Our recent studies of induced pluripotent stem cell (iPSC)-derived dopaminergic neurons with PARK2 knockout (KO) have reported abnormal mitochondrial morphology and function associated with increased oxidative stress and autophagiclysosmal perturbations (Bogetofte et al., 2019b; Okarmus et al., 2020). In mammalian cells it has been shown that dissipation of mitochondrial membrane potential (MMP) leads to recruitment of parkin to mitochondria, followed by their elimination by selective autophagy (mitophagy) (Bertolin et al., 2013). Impairment of mechanisms involved in removal of damaged mitochondria may lead to increased generation of ROS and irrevocable apoptotic cell death, which has been reported for dopaminergic neurons in PD (Hou et al., 2019).

The emergence of metabolomic technologies enables systematic measurement of low-molecular-weight compounds to provide an overview of alterations in metabolic pathways induced by a given perturbation, which could be a gene mutation or infliction of a sporadic disease condition (Forcisi et al., 2015). Metabolomics is therefore useful for studying neurodegenerative disorders as it can reveal disease pathomechanisms and identify potential biomarkers (Andersen et al., 2017). Studies of cerebrospinal fluid (CSF) from patients with PD have identified PD-specific alterations in several metabolic pathways, including polyamine metabolism; the purine, pyruvate, and kynurenine pathways; and redox markers (Andersen et al., 2017; Havelund et al., 2017; Stoessel et al., 2018). Although there is growing interest in the use of metabolomics in PD research, many of these studies do not corroborate each other, possibly due to low sample numbers, clinical heterogeneity, and different analytical approaches (Forcisi et al., 2015).

To our knowledge, no previous studies exist on the metabolomic profile of human iPSC-derived neurons with parkin deficiency. In the present study, we have aimed at demonstrating disease-relevant cellular effects of parkin dysfunction in dopaminergic neurons. To improve our understanding of the parkin-mediated PD phenotype, we describe here the metabolic profile of a PARK2 KO iPSC line compared with its healthy control. We report a meta- bolic signature consisting of 92 compounds of which 51 metabolites significantly distinguish the PARK2 KO neurons from healthy controls. Our study identifies systemic, metabolic pathway alterations that may be caused by primary mitochondrial changes and suggests how disturbances in mitochondrial homeostasis contribute to PD. These findings, which were confirmed in patient-specific iPSC neurons, represent a significant contribution to our understanding of PD pathogenesis and also suggest that PD could be considered a metabolic disorder.

\section{RESULTS}

Metabolomic profiling of PARK2 KO neurons by LCMS

To gain new insight into the role of parkin dysfunction in PD, we applied liquid chromatography-mass spectrometry (LC-MS) metabolomic analysis to compare PARK2 KO neurons with healthy controls. Neurons were generated using a previously described robust differentiation protocol (Bogetofte et al., 2019a, 2019b; Okarmus et al., 2020).

Differentiated cells were collected, and the metabolites were extracted for LC-MS analysis. Of the 92 structurally annotated metabolites, 51 compounds significantly differentiated PARK2 KO neurons from controls (q value <0.05) (Table S1). Principal component analysis (PCA) was used to investigate general interrelationships between the groups, including clustering and outliers among the samples. PCA score plots revealed a clear separation of metabolomes between PARK2 KO neurons and control neurons (Figures 1A and 1B). A clustered analysis heatmap was generated to group related compounds and illustrate the differential profiles (Figure 1C). Following hierarchical cluster analysis, Metabolite Set Enrichment Analysis (MSEA) was performed on detected metabolites to identify which pathways were affected by the distinguishing metabolites (Figure 2A). In parallel, we also utilized the Metabolomic Pathway Analysis (MetPA) module of MetaboAnalyst, which combines results from the pathway enrichment analysis with the pathway topology analysis. A graphical list of the pathways identified and their relative impacts is shown in Figure $2 \mathrm{~B}$. The most important pathways (false discovery rate $<0.05$; impact values $>0.1$ ) included (1) tricarboxylic acid (TCA) cycle; (2) lactate-pyruvate metabolism; (3) glycolysis-gluconeogenesis metabolism; (4) glutathione metabolism; (5) carnitine and

(C) Heatmap of detected metabolomics dataset in the PARK2 KO neurons and control neurons. The heatmap depicts high (red) and low (blue) relative levels of metabolite variation. Individual compounds (vertical axis) are separated using hierarchical clustering (Ward's algorithm) with the dendrogram being scaled to represent the distance between each branch (distance measure: Pearson's correlation). Created using MetaboAnalyst.

See also Table S1. 

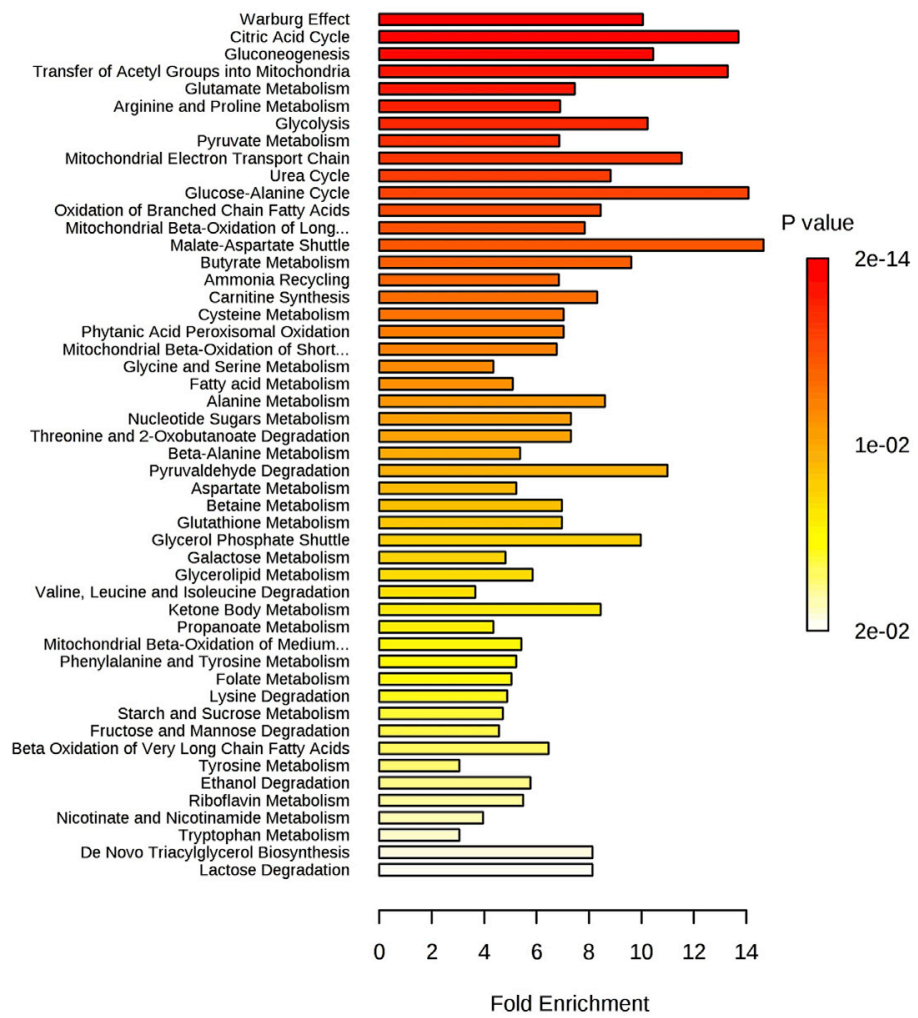

B

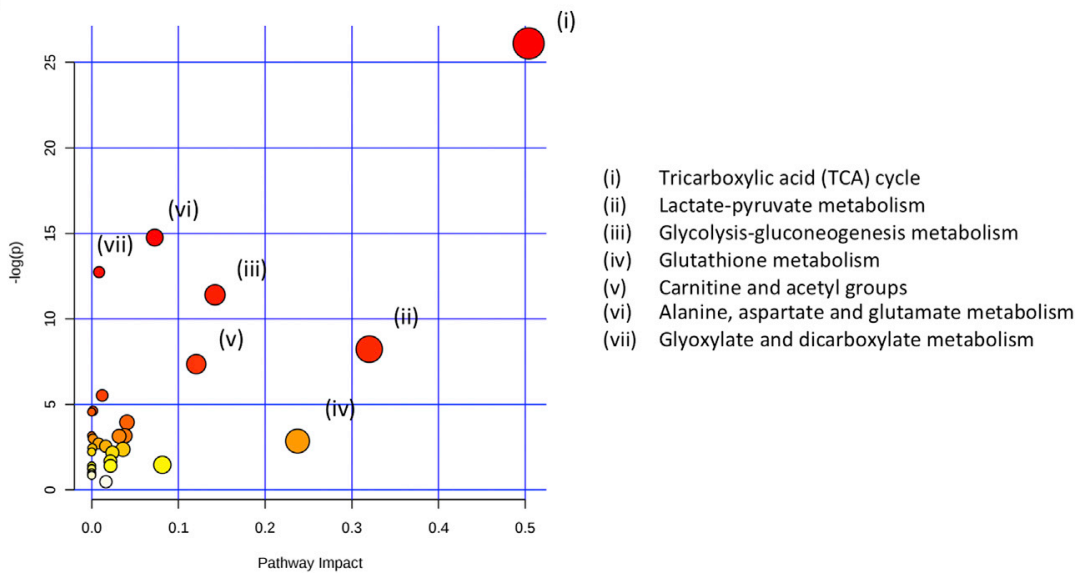

Figure 2. Metabolite-associated pathways (A) Summary plot for the metabolite set enrichment analysis (MSEA) ranked by Holm $p$ value and showing top 50 perturbed pathways (generated by MetaboAnalyst software package). Color intensity (white to red) reflects increasing statistical significance.

(B) Metabolic pathway analysis (MetPA). All the matched pathways are displayed as circles. The color and size of each circle are based on $\mathrm{p}$ value and pathway impact value, respectively. The most impacted pathways with high statistical significance scores are indicated as follows: (i) TCA cycle; (ii) lactate-pyruvate metabolism; (iii) glycolysisgluconeogenesis metabolism; (iv) glutathione metabolism; (v) carnitine and acetyl groups; (vi) alanine, aspartate, and glutamate metabolism; (vii) glyoxylate and dicarboxylate metabolism. Created using MetaboAnalyst. acetyl groups; (6) alanine, aspartate, and glutamate metabolism; and (7) glyoxylate and dicarboxylate metabolism. Taken together, these results demonstrate the capacity of LC-MS to identify a large number of metabolites and metabolic pathways implicated in $\mathrm{PD}$, providing a valuable resource for further investigation.

Increased TCA cycle activity in PARK2 KO neurons TCA, also known as the Krebs cycle, forms a major metabolic hub and is involved in many disease states involving energy imbalance (Chinopoulos, 2013). Using LC-MS metabolomic analysis, we detected seven out of eight TCA cycle intermediates: citrate, isocitrate, $\alpha$-ketoglutarate, succinate, fumarate, malate, and oxaloacetate. The levels of all the detected TCA cycle intermediates, except oxaloacetate, were significantly elevated in the PARK2 KO neurons. The upregulation is presented as fold change compared with healthy control neurons (Figure 3A), and relative abundance was also depicted (Figure 3B). Moreover, we found no difference in glutamine but a significant increase in 
A

\begin{tabular}{|c|c|c|}
\hline $\begin{array}{c}\text { TCA cycle intermediates detected by } \\
\text { metabolomic analysis }\end{array}$ & $\begin{array}{c}\text { Fold change (FC) } \\
\text { PARK2 KO/Control }\end{array}$ & $\begin{array}{c}\text { q-value } \\
\text { Student's } \\
\text { t-test }\end{array}$ \\
\hline$\alpha$-Ketoglutarate & $\mathbf{2 , 9 5 5}$ & 0,001 \\
\hline Succinate & $\mathbf{2 , 5 5 9}$ & 0,001 \\
\hline Fumarate & $\mathbf{1 , 8 7 2}$ & 0,002 \\
\hline Isocitrate & $\mathbf{1 , 6 7 6}$ & 0,018 \\
\hline Malate & $\mathbf{1 , 5 9 7}$ & 0,009 \\
\hline Citrate & $\mathbf{1 , 3 1 8}$ & 0,005 \\
\hline Oxaloacetate & $\mathbf{1 , 3 1 0}$ & 0,162 \\
\hline
\end{tabular}

B
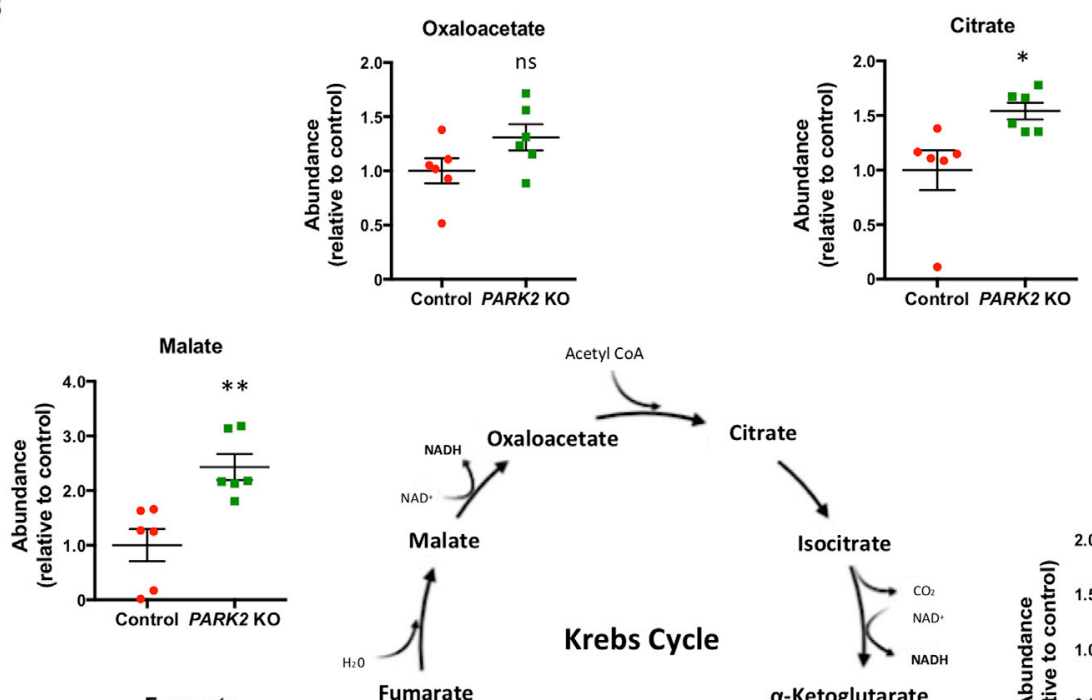

Acetyl COA

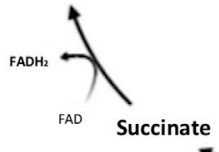

Krebs Cycle
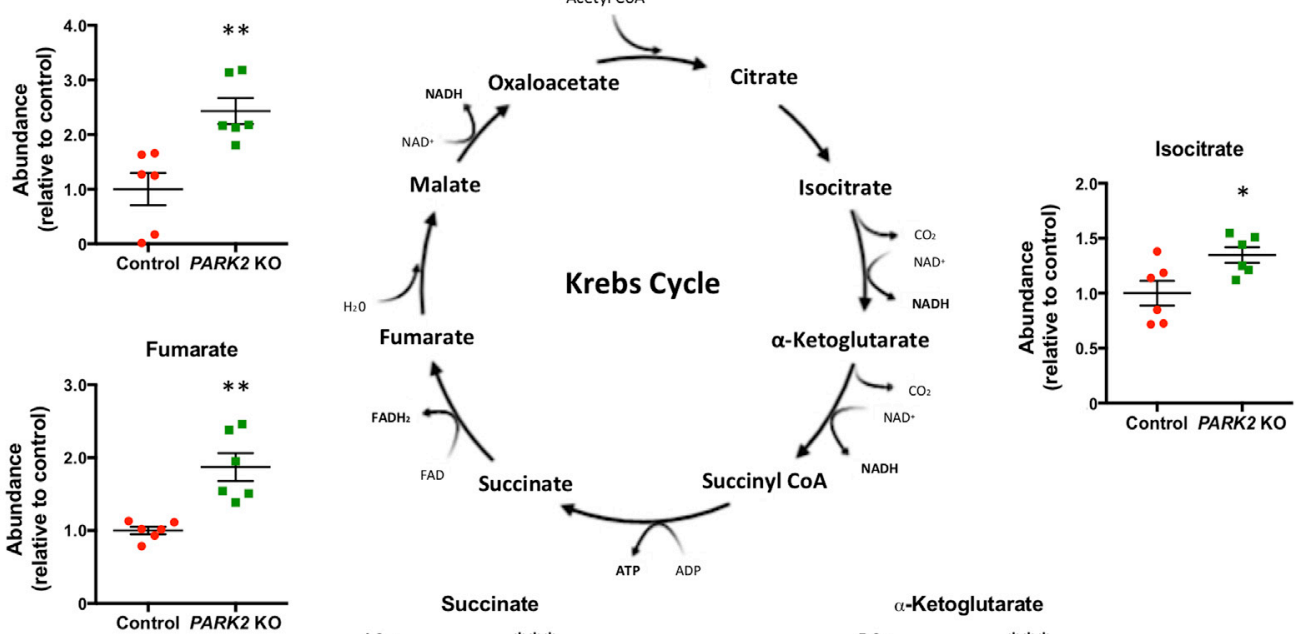

a-Ketoglutarate
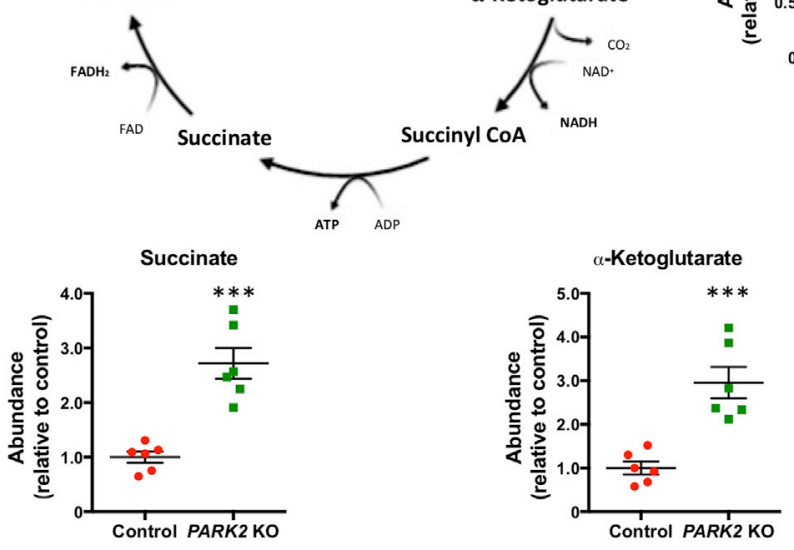

C

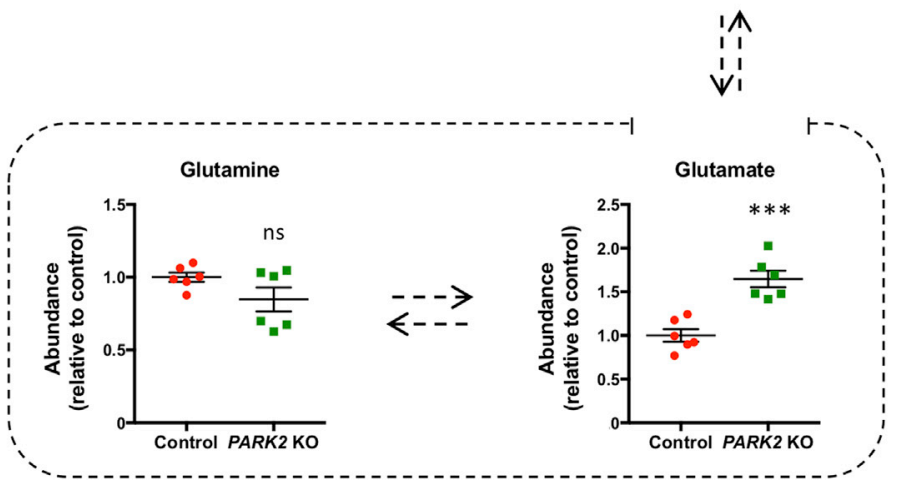

(legend on next page) 
glutamate levels for PARK2 KO neurons (Figure 3C), indicating that the conversion of glutamine to $\alpha$-ketoglutarate via glutamate could serve as the entry of glutamine-derived carbons into the TCA cycle. To investigate the relative contribution of glucose to the TCA cycle we utilized the uniformly labeled glucose $\left(\left[\mathrm{U}_{-}{ }^{13} \mathrm{C}\right]\right.$ glucose). This method enables the observation of $\left[\mathrm{U}_{-}{ }^{13} \mathrm{C}\right]$ glucose conversion to downstream metabolites and determination the fraction of specific metabolites derived from consumed glucose (Figure 4A). Analysis of the labeling patterns during $2 \mathrm{~h}$ of incubation with ${ }^{13} \mathrm{C}$-labeled glucose showed an increase in the percentage of total labeling of citrate, $\alpha$-ketoglutarate, succinate, and fumarate (Figures $4 \mathrm{~B}-4 \mathrm{E}$ ), consistent with the previously observed accumulation of TCA cycle intermediates (Figures 3A and 3B). Similarly, the labeling of lactate and glutamate was higher for PARK2 KO neurons compared with controls (Figures $4 \mathrm{~F}$ and $4 \mathrm{G}$ ). The mass of the individual labeled glucose carbons (the individual isotopic distribution for each isotopomer) of key metabolites is presented in Figure S1. Overall, these findings suggest that parkin deficiency promotes the metabolic flux of the TCA cycle, in which both glucose- and glutamine-derived carbons contribute to the observed accumulation of TCA intermediates.

Mitochondrial membrane potential, mitochondrial ultrastructure, and ATP levels are perturbed in PARK2 KO neurons

To study the role of parkin in mitochondrial maintenance and function, mitochondrial membrane potential (MMP) was monitored in PARK2 $\mathrm{KO}$ and control neurons using tetramethylrhodamine ethyl ester (TMRE), while FCCP treatment served as a negative control. When neurons were loaded with TMRE and examined by fluorescence microscopy, the PARK2 KO neurons appeared to have diminished MMP (Figure $4 \mathrm{H}$ ). We verified this by a fluorescence plate reader-based quantification of the TMRE signal, which showed similarly reduced MMP (Figure 4I). In addition to the functional mitochondrial analyses, comparative transmission electron microscopy (TEM) revealed clear changes in mitochondrial morphology in the PARK2 KO neurons. Mitochondria in control neurons displayed a typical oval profile with well-organized cristae, whereas mitochondria of PARK2 KO neurons were swollen and exhibited aberrant cristae, disorganized inner membrane, and non-dense ma- trix (Figure 4J). Based on these morphological observations, mitochondria were categorized into two groups: normal (with well-organized cristae) and abnormal (swollen with irregular cristae). Although quantification of mitochondrial number relative to cytoplasm showed no significant differences in total mitochondria per cell between PARK2 $\mathrm{KO}$ and control neurons, there was a significant difference in the number of abnormal mitochondria (Figure $4 \mathrm{~K}$ ). Since changes to the organization of the mitochondrial internal membrane are known to regulate mitochondrial respiratory function (Errea et al., 2015), the complexity of mitochondrial cristae was analyzed using the ratio between the mitochondrial cristae perimeter and the external perimeter. This ratio was significantly decreased in PARK2 KO neurons compared with control (Figure $4 \mathrm{~L}$ ), indicating loss of cristae complexity. Reduction in MMP and disruption of mitochondrial ultrastructure, including cristae complexity in the PARK2 KO neurons, may suggest disturbances in the electron transport chain, which is crucial for ATP production (Nsiah-Sefaa and McKenzie, 2016). Therefore, to test energy production in the generated neurons, we measured the cellular ATP content at steady state. This was significantly decreased in neurons carrying PARK2 $\mathrm{KO}$ compared with control neurons (Figure $4 \mathrm{M}$ ), indicating an energy deficiency. Moreover, we have previously examined the mitochondrial respiration of PARK2 KO neurons and demonstrated that both the basal oxygen consumption rate (OCR) and ATP production were reduced when respiring on lactate. Upon incubation with glucose, the OCR of PARK2 KO neurons was similar to that of controls, but the basal extracellular acidification rate (ECAR), an indicator of glycolysis, was reduced (Bogetofte et al., 2019b).

Dysregulation of glucose metabolism and lactate accumulation in PARK2 KO neurons

The detected TCA perturbations and ATP depletion correlated well with our earlier findings of decreased levels of a number of proteins of importance for energy metabolism (Bogetofte et al., 2019b). This prompted us to perform a joint protein-metabolite pathway analysis. Particularly in the glycolytic pathway, we found a high occurrence of both proteins and metabolites that were significantly dysregulated, including two important glycolytic enzymes, pyruvate dehydrogenase kinase (PKM) and lactate dehydrogenase (LDH), which were significantly reduced in the

Figure 3. Metabolomic profile of the TCA cycle

(A) Table listing fold change for detected TCA intermediates in metabolomics analysis.

(B) Graphical overview of TCA cycle indicating increased levels of its intermediates (citrate, isocitrate, $\alpha$-ketoglutarate, succinate, fumarate, malate, and oxaloacetate) in the PARK2 KO neurons.

(C) The levels of glutamine and glutamate in PARK2 KO neurons compared with healthy controls and their conversion to $\alpha$-ketoglutarate. Data presented as mean \pm SEM, data from three independent differentiations, significant differences are indicated by * $p<0.05$; ${ }^{* *} p<0.01 ;{ }^{* *} p<0.001$; and ns, not significant, Student's t test. 
A

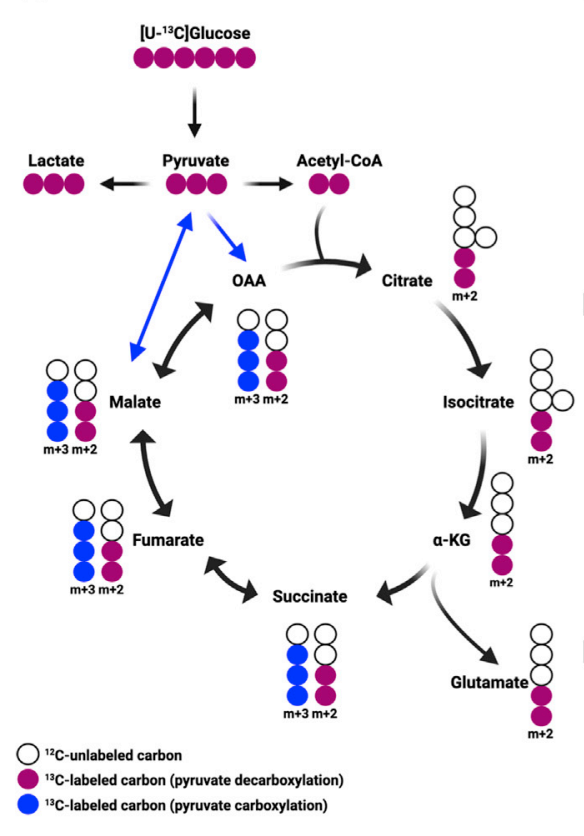

B

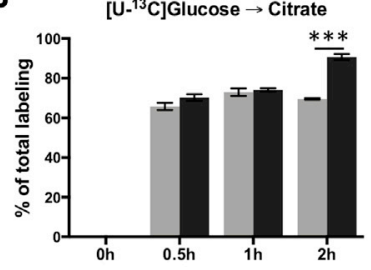

D

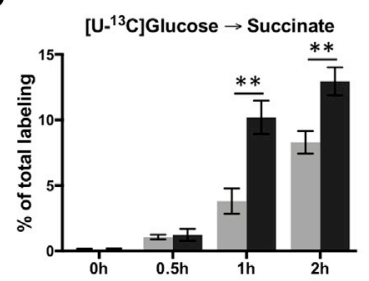

$\mathbf{F}$

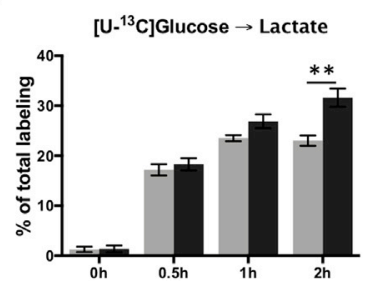

H

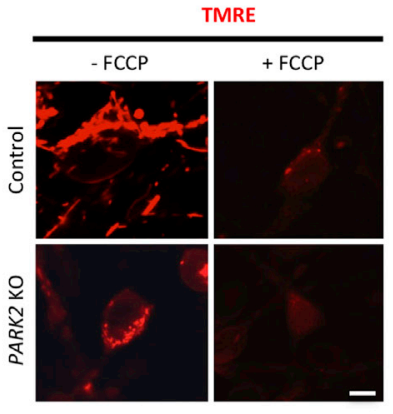

$\mathbf{K}$

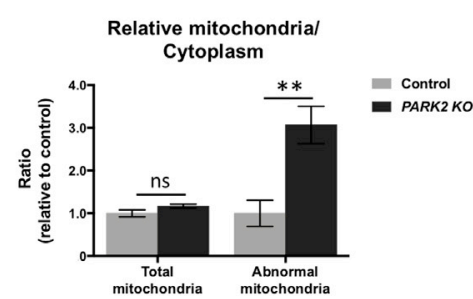

I

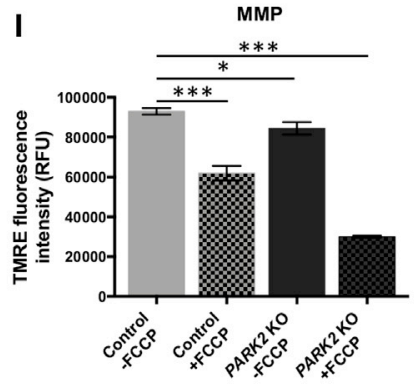

$\mathbf{L}$

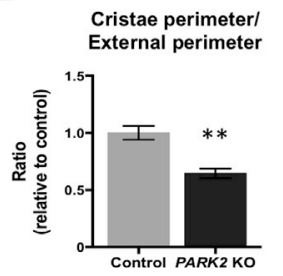

C

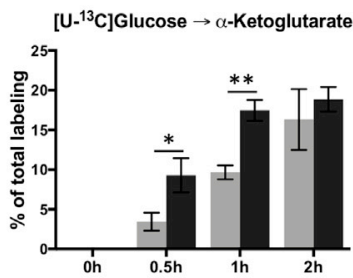

E

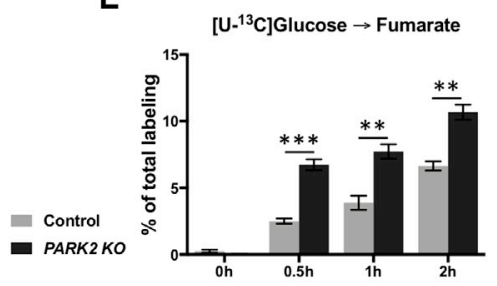

G

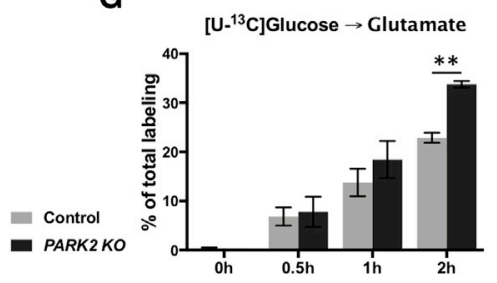

$\mathbf{J}$

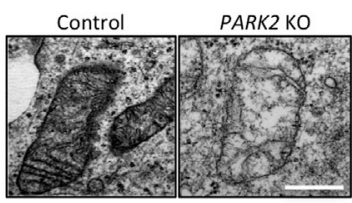

M

ATP production

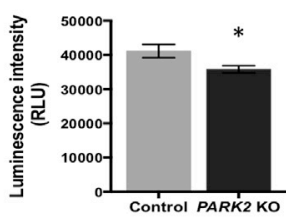

Figure 4. TCA cycle, MMP, mitochondrial ultrastructure, and cellular ATP levels are perturbed in PARK2 KO neurons (A) $\left[\mathrm{U}-{ }^{13} \mathrm{C}\right] \mathrm{Glucose}$ pathway showing labeling patterns of downstream metabolites. White circles represent ${ }^{12} \mathrm{C}$-unlabeled carbons, purple circles depict ${ }^{13} \mathrm{C}$-labeled carbons from pyruvate decarboxylation, whereas blue circles display ${ }^{13} \mathrm{C}$-labeled carbons from pyruvate carboxylation. $\alpha$-KG, $\alpha$-ketoglutarate; OAA, oxaloacetate. Created using BioRender.

(B-G) Percentage of total labeling (expressed as the sum of the individual isotopomers normalized to the $m+0$ ) of (B) citrate, (C) $\alpha$-ketoglutarate, (D) succinate, (E) fumarate, (F) lactate, and (G) glutamate in PARK2 K0 and control neurons incubated for 0.5, 1, and $2 \mathrm{~h}$ with $24.2 \mathrm{mM}\left[\mathrm{U}-{ }^{13} \mathrm{C}\right] \mathrm{glucose}$. Data are presented as mean $\pm \mathrm{SEM}$, data from three independent differentiations, significant differences are indicated by ${ }^{*} p<0.05,{ }^{* *} p<0.01,{ }^{* *} p<0.001$, multiple $t$ test followed by Holm-Sidak's test for multiple comparisons. The data of these metabolites for the individual ${ }^{13} \mathrm{C}$-labeled carbons are compiled in Figure S1.

(H) Differentiated neurons were loaded with mitochondrial membrane indicator TMRE (50 nM). Changes in fluorescence were measured without (left) and with (right) application of the membrane potential uncoupler, FCCP (negative control). Scale bar: $10 \mu \mathrm{m}$. 
PARK2 KO neurons (Figure S2). When we compared the levels of glycolytic intermediates detected by the metabolomic analysis, we found that glucose levels were significantly decreased in the PARK2 KO neuronal cultures (Figure $5 \mathrm{~A}$ ). Further intermediates of the glycolysis pathway that were detected by LC-MS were D-glucose-6-phosphate, fructose-1,6-bisphosphate, and the end product pyruvate. Although no difference in the level of D-glucose-6-phosphate was found (Figure $5 \mathrm{~B}$ ), we detected a significant increase in the level of fructose-1,6-bisphosphate, an allosteric activator of glycolysis, in the PARK2 KO neurons (Figure 5C). Importantly, the abundance of pyruvate was reduced in the PARK2 $\mathrm{KO}$ neurons alongside a highly elevated level of lactate. As lactate is a downstream byproduct of pyruvate in the anaerobic glycolysis, this suggests perturbations in the glycolytic pathway and upregulation of lactate (Figures 5D-5F). To confirm our observations, we also determined lactate enzymatically. Consistent with our metabolomic measurements, we found that both intra- and extracellular lactate levels were significantly increased in the PARK2 KO neuronal cultures compared with controls (Figure 5G). Finally, we determined glucose uptake by measuring the uptake of the fluorescent glucose analogue, 2-deoxy-D-glucose(2-DG). No significant difference in glucose uptake was found between the two neuronal populations (Figure $5 \mathrm{H}$ ), indicating that glucose uptake was not responsible for driving the glycolytic changes observed for the PARK2 KO neurons.

Accumulation of short- and long-chain carnitines in PARK2 KO neurons

To further investigate changes in energy-related metabolic pathways, we examined carnitine homeostasis. The carnitine shuttle (Figure 5I) represents a mechanism by which long-chain fatty acids, impermeable to the mitochondrial membranes, are transported into the mitochondrial matrix for $\beta$-oxidation and energy production (Longo et al., 2016). The level of free L-carnitine, which is required for mitochondrial entry of long-chain fatty acids, was significantly reduced in the PARK2 $\mathrm{KO}$ neurons compared with isogenic controls (Figure 5J). In contrast, there was a marked elevation in short- and long-chain acylcarnitines (Figures $5 \mathrm{~K}$ and 5L). More specifically, we found an increase in C3-carnitine (propionyl-L-carnitine) and C4-carnitine (O-butanoylL-carnitine) as well as unsaturated fatty acid carnitine conjugate profile, palmitoyl-L-carnitine (C16), suggesting decreased lipid $\beta$-oxidation in PARK2-deficient neurons.

\section{PARK2 KO neurons display differential oxidative stress-associated metabolic parameters}

LC-MS metabolomic profiling suggested that oxidative stress may also be linked to neuronal complications associated with PD. Although the levels of reduced glutathione (GSH) were not significantly changed in the PARK2 KO neurons (Figure 6A), the level of oxidized glutathione (GSSG) was increased (Figure 6B). Accordingly, PARK2 KO neurons showed a marked decrease in the GSH/GSSG ratio (Figure $6 \mathrm{C}$ ), suggesting elevated oxidative stress. In addition, the $\mathrm{NAD}^{+} / \mathrm{NADH}$ ratio, which plays a role in preventing cellular oxidative damage, was imbalanced in the PARK2 KO neurons (Figure 6D); this was verified with a bioluminescence assay (Figure 6E). In line with the present findings, our previously reported proteomic analysis identified decreased levels of proteins related to superoxide radical degradation and metabolism of ROS, including superoxide dismutase (SOD) 1, catalase, and DJ-1 (Figure 6F) (Bogetofte et al., 2019b). As imbalance in energy metabolism combined with defective oxidative stress defense is associated with increased ROS production (Dias et al., 2013), we evaluated ROS levels in the PARK2 KO neurons and healthy controls. Basal cytosolic ROS production was significantly higher in the PARK2 KO neurons than in the control cells (Figure 6G), which was also reported in our recent study (Okarmus et al., 2020). Overall, we found that oxidative stress was aggravated in the PARK2 KO neurons.

Metabolomic analysis of patient-specific iPSC-derived neurons supports the key findings identified in PARK2 KO neurons

In order to validate the parkin-induced metabolic disturbances observed for our isogenic cell lines, we performed a separate series of metabolomic experiments using a set of PARK2 and control patient iPSC lines. The utilized cell lines (Figure S3, Table S2) were differentiated and

(I) Graph presenting TMRE intensity from plate reader analysis. Data presented as mean \pm SEM, data from independent experiments, significant differences are indicated by ${ }^{*} p<0.05,{ }^{* *} p<0.001$, one-way ANOVA followed by Dunnett's post hoc test for multiple comparisons.

(J) TEM pictures presenting mitochondria in control and PARK2 KO neurons. Scale bar: $500 \mathrm{~nm}$.

(K) Quantification of mitochondria/cytoplasm ratios showing the relative abundance of total and abnormal mitochondria in PARK2 KO neurons.

(L) The ratio between the internal cristae perimeter and the external perimeter.

(M) Total cellular ATP level determined using a luciferase assay kit in PARK2 KO neurons. Data are presented as mean \pm SEM, data from three independent differentiations, significant differences are indicated by ${ }^{*} p<0.05, ;{ }^{*} p<0.01$; ns, not significant; multiple t test followed by Holm-Sidak's test for multiple comparisons $(K)$ or Student's t test $(L, M)$. 
A

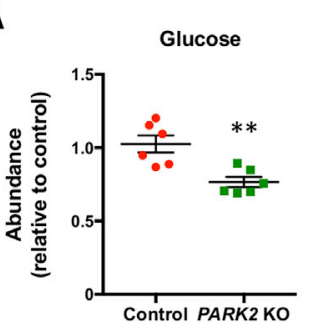

D

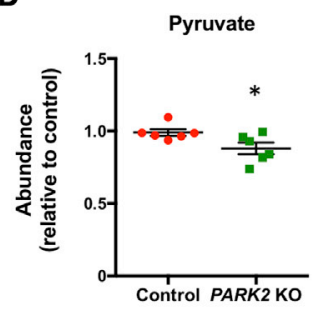

B D-Glucose-6-phosphate

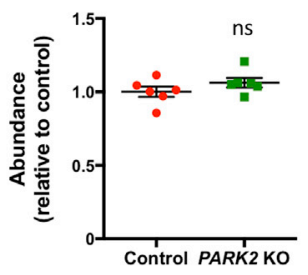

E

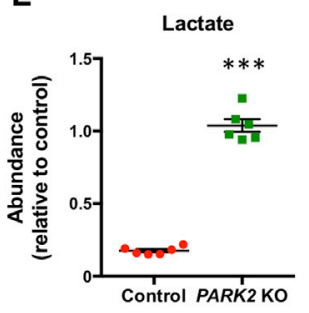

C Fructose-1,6-bisphosphate

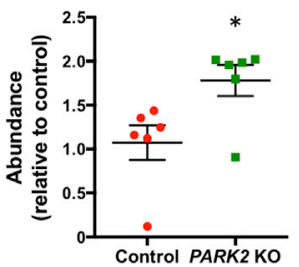

$\mathbf{F}$

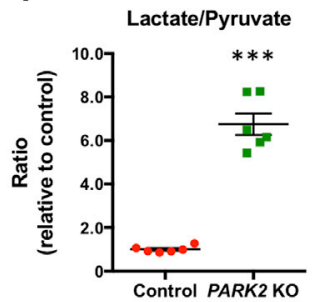

H Glucose uptake

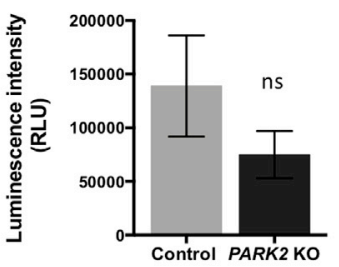

J

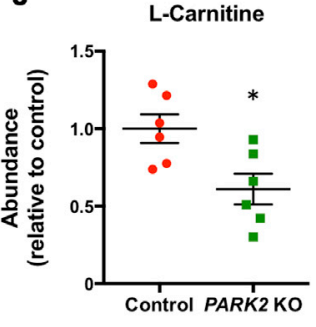

$\mathbf{K}$
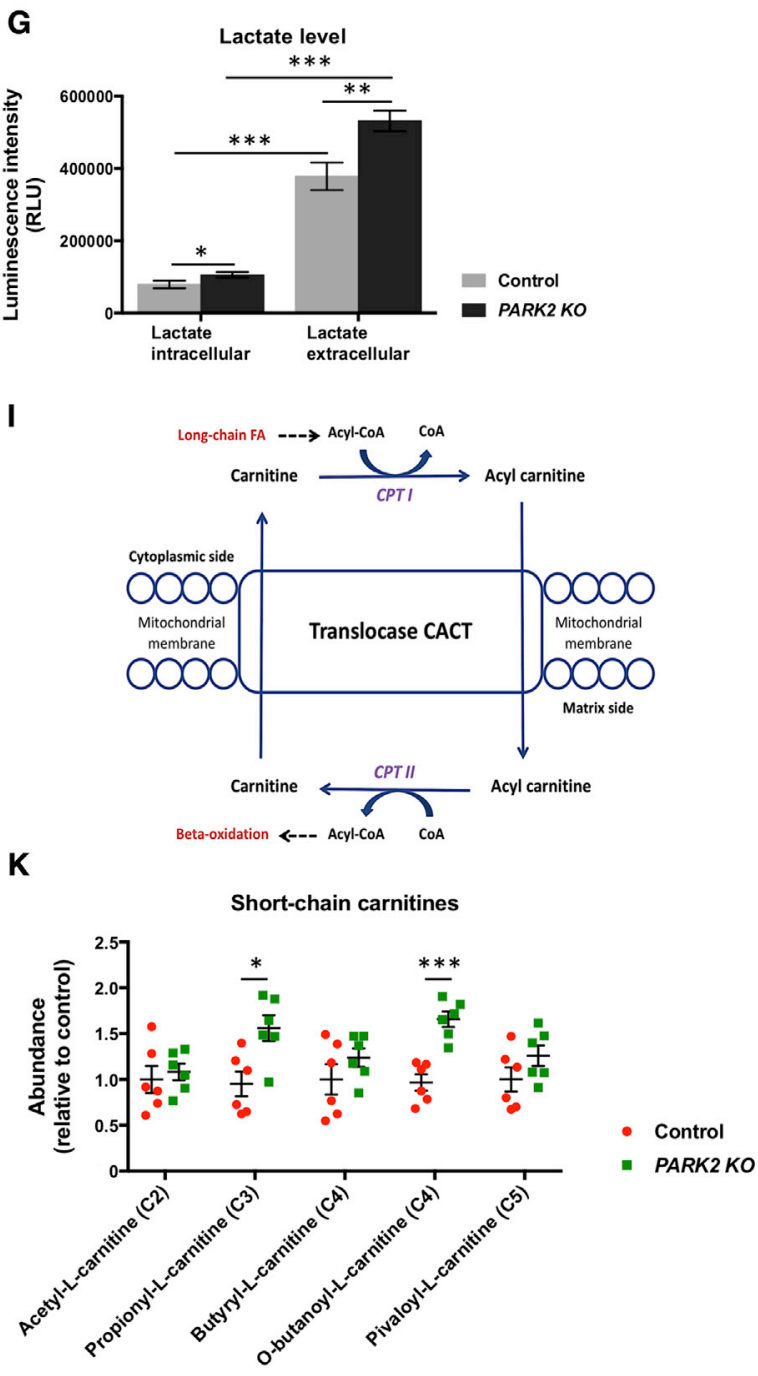

$\mathbf{L}$

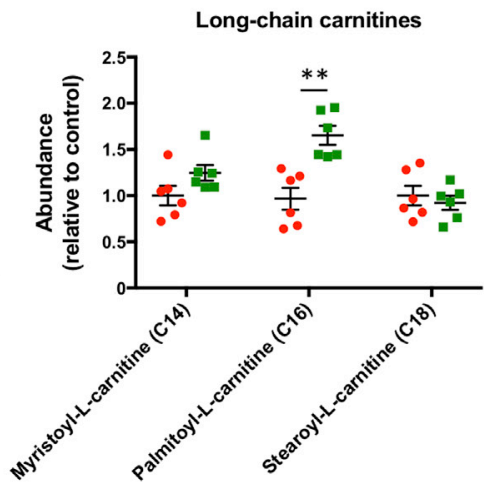

(legend on next page) 
characterized according to well-established protocols (Grünewald et al., 2010; Haenseler et al., 2017; Lang et al., 2019). In agreement with data obtained for the isogenic iPSC lines, we found mitochondrial and energy-related changes in the form of elevated levels of citrate, succinate, malate, and glutamate (Figures 7A-7D) and reduced levels of pyruvate and L-carnitine (Figures 7E and 7F). The changes associated with oxidative stress were expressed by a decrease in the GSH/GSSG ratio and $\mathrm{NAD}^{+}$abundance (Figures $7 \mathrm{G}$ and $7 \mathrm{H}$ ). Data for the individual cell lines are compiled in Figure S4. Overall, these results indicate that the observed parkin-induced metabolic changes are not specific to the isogenic PARK2 $\mathrm{KO}$ and control cells, but are reproducible in PARK2 patient-derived neurons.

\section{DISCUSSION}

Metabolomic analysis is a powerful tool for investigating global changes in cellular metabolism. Several metabolomic studies have been performed on plasma, CSF, or blood samples from PD patients with the aim of identifying potential biomarkers (LeWitt, 2012; Trezzi et al., 2017). To our knowledge, no data have previously been generated on the metabolomic profile of human iPSC-derived neurons with parkin deficiency. We report here that loss of parkin function induces metabolic dysregulation. We show that PARK2 mutation in human iPSC-derived neurons affected their general metabolome, likely caused by altered mitochondrial and energy homeostasis combined with increased oxidative stress and decreased anti-oxidative response. Interestingly, our results are consistent with data obtained by metabolomic analysis of serum from PARK2 patients (Okuzumi et al., 2019), showing significantly higher levels of fatty acid metabolites and oxidized lipids, and significantly lower levels of antioxidants, similar to those of idiopathic PD patients. Considering the alterations in common metabolic pathways observed for PARK2 iPSC-derived neurons, serum from PARK2 and idiopathic PD subjects, parkin dysfunction may play important roles in the pathomechanism of both conditions.
Energy metabolism in the central nervous system is anticipated to be essential for neuronal health; therefore, impaired energy metabolism plays an important role in the pathogenesis of PD (Blaszczyk, 2018). Most of the cellular energy in healthy cells is produced by OXPHOS from the energy precursors produced in the TCA cycle in mitochondria (Bratic and Trifunovic, 2010). Interestingly, our results showed accumulation of metabolites associated with the TCA cycle in the PARK2 KO neurons. As the rate of the TCA cycle is regulated primarily by the concentration of ATP and NADH (Fernie et al., 2004), this accumulation of TCA intermediates in PARK2 KO neurons might be a consequence of the detected low ATP and high NADH levels. Alterations in levels of TCA cycle metabolites have been reported previously in relation to PD. For instance, an increased malate concentration was found in plasma (Willkommen et al., 2018), and an increased citrate concentration in the CSF (Nagesh Babu et al., 2018) of PD patients. In contrast, decreased levels of several TCA metabolites (citrate, malate, succinate, and isocitrate) have also been detected in brains from PD patients (Gibson et al., 2003). These inconsistencies may be due to the different types of samples being analyzed (neuronal cell cultures, biofluids, brain tissues) or technical variations in sample matrix, sampling, storage, and measurement methods. Moreover, similarly to our results, an increase in concentrations of TCA intermediates was reported in samples from patients with mitochondrial disorders (Delaney et al., 2017). Even if existing literature is slightly divergent, these data point overall to TCA dysregulation resulting in mitochondrial and energy perturbations involved in PD. Data on ${ }^{13} \mathrm{C}$-labeled glucose confirmed our other results and indicated that TCA metabolites are mainly labeled as $m+2$ and $m+3$, through both pyruvate decarboxylation and carboxylation. Overall, our findings show that both glucose- and glutamine/glutamate-derived carbons contribute to the observed accumulation of TCA intermediates.

Given the perturbed TCA cycle observed in the PARK2 KO neurons, we further investigated mitochondrial function and found reduced levels of ATP, a critical parameter

Figure 5. PARK2 K0 neurons display disturbances in glucose, lactate, and carnitine metabolism

(A-F) Abundance as measured by LC-MS of (A) glucose and further intermediates: (B) D-glucose-6-phosphate and (C) fructose-1,6-bisphosphate in PARK2 K0 and control neurons. Comparison of the levels of (D) pyruvate, (E) lactate, and (F) ratio between lactate and pyruvate in the PARK2 KO neurons compared with healthy controls.

$(\mathrm{G}$ and $\mathrm{H})$ Levels of $(\mathrm{G})$ intracellular and extracellular lactate and $(\mathrm{H})$ glucose uptake in PARK2 K0 neurons measured using bioluminescent assays. Data are presented as mean \pm SEM, data from three independent differentiations, significant differences are indicated by * $p<0.05$; ${ }^{* *} \mathrm{p}<0.01 ;{ }^{* * *} \mathrm{p}<0.001$; ns, not significant, Student's t test (A-F and H) or two-way ANOVA followed by Tukey's post hoc test for multiple comparisons (G). See also Figure S2.

(I) Schematic representation of the carnitine shuttle.

$(\mathrm{J}-\mathrm{L})$ Levels of $(\mathrm{J})$ free $\mathrm{L}$-carnitine, $(\mathrm{K})$ short-chain, and $(\mathrm{L})$ long-chain acylcarnitines in the PARK2 K0 and control neurons as measured by LC-MS. Data are presented as mean \pm SEM, data from three independent differentiations, significant differences are indicated by * $p<0.05$, ${ }^{* *} p<0.01$, and ${ }^{* *} p<0.001$, Student's t test $(\mathrm{J})$ or multiple $t$ test followed by Holm-Sidak's test for multiple comparisons (K and L). 
A

GSH

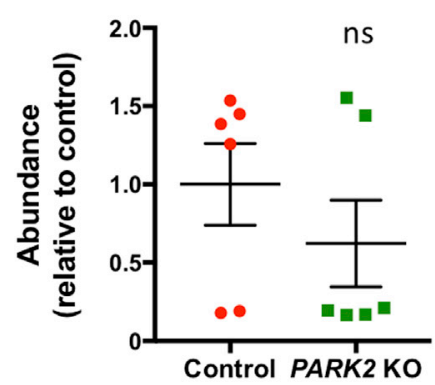

D

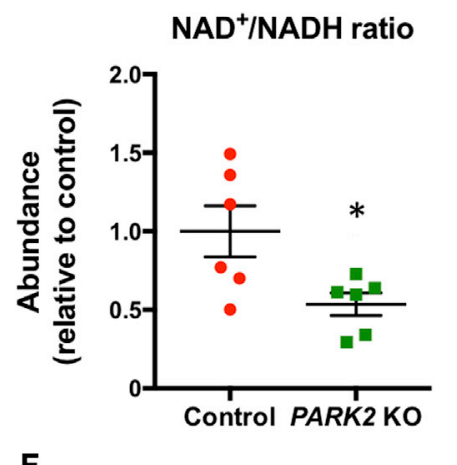

B

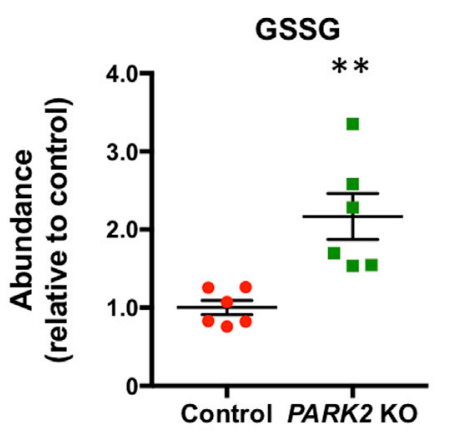

E

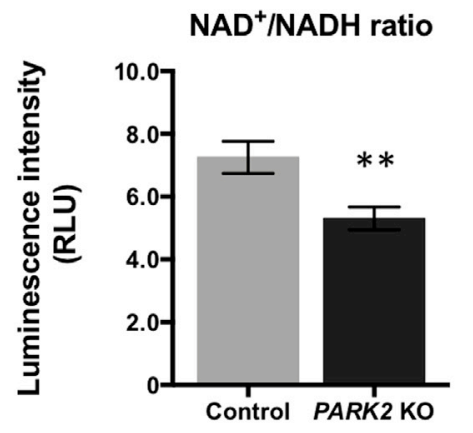

C

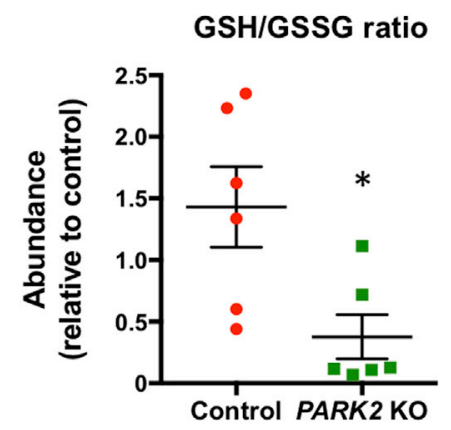

G

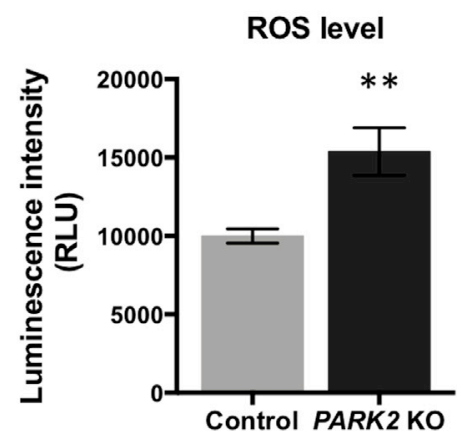

$\mathbf{F}$

\begin{tabular}{|c|c|c|}
\hline $\begin{array}{c}\text { Proteins detected by } \\
\text { proteomic analysis }\end{array}$ & $\begin{array}{c}\text { Fold change (FC) } \\
\text { PARK2 KO/Control }\end{array}$ & $\begin{array}{c}\text { q-value } \\
\text { Student's } \\
\text { t-test }\end{array}$ \\
\hline Protein deglycase DJ-1 & $\mathbf{0 , 8 2}$ & 0,022 \\
\hline Superoxide dismutase 1 (SOD1) & $\mathbf{0 , 6 4}$ & 0,033 \\
\hline Catalase & $\mathbf{0 , 3 4}$ & 0,000 \\
\hline
\end{tabular}

Figure 6. Increased oxidative stress and decreased oxidative stress defense in PARK2 KO neurons (A-C) Levels of (A) GSH, (B) GSSG, and (C) the GSH/GSSG ratio revealed by metabolomic analysis.

(D) NAD $/ \mathrm{NADH}$ ratio measured by metabolomics and (E) verified using a bioluminescence assay.

(F) Table listing the earlier identified proteins related to oxidative stress defense with the ratio of proteins levels in PARK2 K0 neurons compared with controls (Bogetofte et al., 2019b).

(G) Levels of cellular ROS production. Data are presented as mean \pm SEM, data from three independent differentiations, significant differences are indicated by ${ }^{*} p<0.05 ;{ }^{*} p<0.01 ; n s$, not significant; Student's t test.

of cellular energy status. These data were strengthened by the observation of aberrant mitochondrial cristae ultrastructure and reduced MMP in the PARK2 KO neurons. In line with our observations, Imaizumi et al. (2012) reported an abnormal mitochondrial morphology for PARK2 iPSC-derived neurons. They detected accumulation of mitochondria with a highly electron-dense matrix and swollen cristae. The inner membrane structures are crucial for ATP production due to maintaining MMP, which provides the driving force for ATP synthesis (Dimroth et al.,
2000). A few other studies, including our own, of PD patient iPSC-derived neurons with PARK2 mutation have also reported aberrant mitochondrial morphology and function (Bogetofte et al., 2019b; Chung et al., 2016; Ren et al., 2015), and several prior studies have addressed changes in energy metabolism associated with PD. The fall in ATP has been reported in different experimental PD models, such as MPTP mouse model (Meredith and Rademacher, 2011) or skin fibroblasts isolated from PD patients (Haylett et al., 2016). 
A

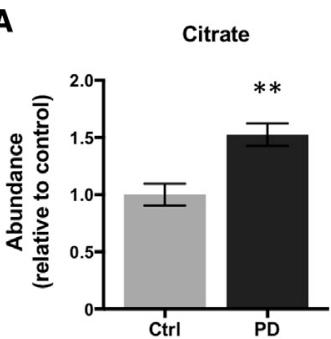

D

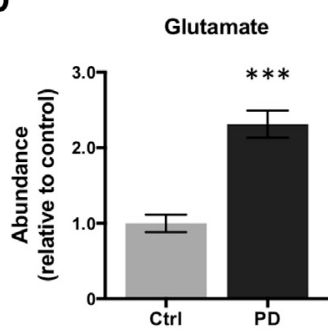

B

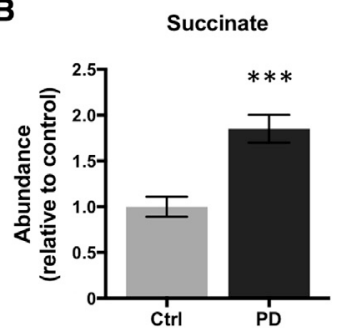

E

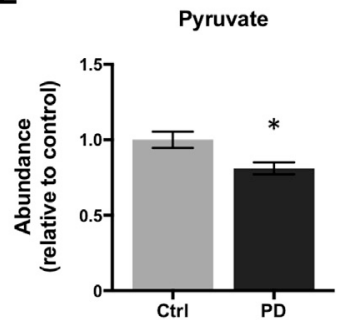

C

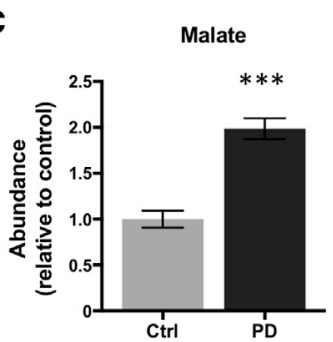

$\mathbf{F}$

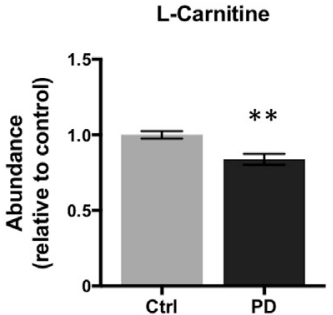

G

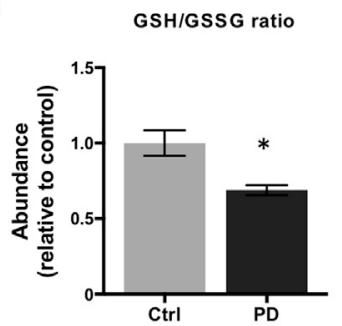

H

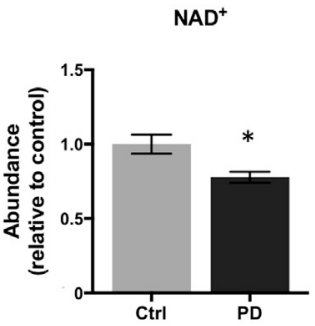

Figure 7. Validation of PARK2 KO metabolic changes using patient-specific iPSC-derived neurons

(A-F) Data from PARK2 patient iPSC-derived neurons that validate the phenotype of PARK2 K0 cells by displaying mitochondrial and energy-related alterations in form of elevated levels of $(A)$ citrate, $(B)$ succinate, $(C)$ malate, and (D) glutamate, and reduced levels of (E) pyruvate, (F) L-carnitine.

$(\mathrm{G}$ and $\mathrm{H})$ The changes associated with oxidative stress are indicated by a decrease in $(\mathrm{G})$ the GSH/GSSG ratio and $(H)$ NAD ${ }^{+}$abundance. Data are presented as mean $\pm S E M$, pooled data from independent cell lines, significant differences are indicated by * $p<0.05,{ }^{* *} p<0.01$, $* * * p<0.001$, Student's t test.

See Figures S3 and S4 and Table S2 for additional data.

The altered energy and mitochondrial metabolism described in the present study verifies and complements our earlier study in which we reported mitochondria-specific proteomic and phospho-proteomic changes caused by PARK2 KO. We found several mitochondrial protein changes combined with disturbances in mitochondrial morphology and the mitochondrial respiration function, among others. In addition, we previously reported changes in mitochondrial respiration and demonstrated that PARK2 KO neurons have a significantly decreased ability to respire using lactate as a substrate (Bogetofte et al., 2019b).

Glycolysis is a process in which glucose is converted into pyruvate or lactate depending on whether it occurs under aerobic or anaerobic conditions. The regulation of glycolysis is complex and occurs at multiple stages (Berg et al., 2002). In the present study, we detected dysregulation of glucose metabolism in PARK2 KO neurons at various levels of the glycolytic pathway. We found a significant decrease in levels of both glucose (the initial substrate of glycolysis) and pyruvate (the end product of aerobic glycolysis), which was not driven by changes in glucose uptake. This, together with the reduced levels of enzymes involved in the glycolytic regulation, detected by proteomic analysis, is indicative of a decrease in glycolytic capacity in PARK2 KO neurons. These results are in line with our previous data showing that PARK2 KO causes a significant decrease in the basal ECAR (glucose as a substrate), which strongly indicates a decrease in anaerobic glycolysis (Bogetofte et al., 2019b). Fructose1,6-bisphosphate, the level of which was elevated in PARK2 KO cells, is one of the most significant regulators of glycolysis. This phosphorylated fructose sugar is an intermediate of the glycolysis pathway and provides feedforward stimulation as an allosteric activator of two key enzymes of glycolysis: phosphofructokinase (PFK) and pyruvate kinase (PK) (Jurica et al., 1998). Increased level of fructose1,6-bisphosphate may represent a compensatory mechanism since levels of both key enzymes were significantly reduced in the PARK2 KO neurons.

In the present study, we found increased lactate and decreased pyruvate levels for PARK2 KO neurons compared with healthy controls. This can be explained by the fact that pyruvate enters the TCA cycle via carboxylation and decarboxylation and is metabolized into lactate by LDH. Further, the significantly elevated ratio between lactate and pyruvate in PARK2 KO neurons can be an indicator of alterations in protein degradation (Crie and Wildenthal, 1980) as well as decreased lactate clearance (Andersen et al., 2013). In addition, a buildup 
of lactic acid can cause suppression of glycolytic enzymes (Leite et al., 2011), which to some extent may explain their low level in PARK2 $\mathrm{KO}$ neurons. An increase in lactate levels may be a result of mitochondrial dysfunction promoting the conversion of pyruvate to lactate, which has been reported for mice (Ross et al., 2010). However, our recent paper showed that the basal OCR was significantly reduced in PARK $2 \mathrm{KO}$ neurons respiring on lactate, which is indicative of impaired lactate-supported respiration (Bogetofte et al., 2019b). It is worth considering that the changed lactate-pyruvate ratio found for PARK2 KO neurons may be caused by buildup of astrocyte-derived lactate. This assumption is meaningful since there is evidence that astrocyte-derived lactate is an important energy source of the human brain that also may serve as a neuroprotective factor in pathological conditions (Jourdain et al., 2016). Overall, the present metabolomic findings are consistent with our previously reported proteomic data, suggesting impaired glycolysis and disturbances in lactate metabolism (Bogetofte et al., 2019b). In general, our data show that the combination of proteomics and metabolomics represents a very valuable platform for better understanding of energy metabolism and disease mechanisms.

Carnitine also plays a critical role in energy production due to transporting long-chain fatty acids into the mitochondria where they can be oxidized (Sharma and Black, 2009). The reduction in L-carnitine level and the elevation in some short- and long-chain acylcarnitines in the PARK2 KO neurons reflect an underling disturbance of mitochondrial fatty acid $\beta$-oxidation, suggesting an impaired lipidhandling capacity (Longo et al., 2016). The observed accumulation of short- and long-chain fatty acid carnitine conjugates and decreased L-carnitine also suggest defects in the carnitine shuttle and hence impaired mitochondrial import of fatty acids. These defects may lead to detrimental effects on ATP production as the import of long-chain fatty acids into the mitochondria is rate limiting for lipid $\beta$-oxidation (Mihalik et al., 2010). Increased levels of saturated chain acylcarnitines have been reported in patients with metabolic disorders (Mihalik et al., 2010), although another study showed the opposite: a reduction in acylcarnitines (Saiki et al., 2017). Further studies are warranted in this matter due to the conflicting literature, but all these results demonstrate energy dysregulation of fatty acid oxidation.

It has been suggested that fatty acid metabolism plays a role in the maintenance and survival of nigrostriatal dopaminergic neurons, and thus alterations in lipid biology may lead to PD (Bousquet et al., 2011). Consistent with this hypothesis, our metabolomic analysis of PARK2 KO iPSC-derived dopaminergic neurons revealed a decrease in lysolipid levels and an increase in fatty acid-carnitines. Moreover, significantly elevated levels of fatty acids have been described in serum samples from PARK2 patients (Okuzumi et al., 2019). Indeed, parkin has been reported to regulate systemic lipid homeostasis and fat uptake via ubiquitin-mediated stabilization of the lipid transporter CD36 (Kim et al., 2011), suggesting an important role of disturbed lipid homeostasis in PD.

Oxidative stress is a condition associated with an increased rate of cellular damage and is manifested by the excessive production of ROS with insufficient or defective antioxidant defense systems (Nita and Grzybowski, 2016). Oxidative stress causes profound alterations in various biological structures, including cellular membranes, lipids, proteins, and nucleic acids (Therond, 2006). We have found substantially decreased levels of several antioxidant enzymes (Bogetofte et al., 2019b) as well as increased mitochondrial ROS levels in the PARK2 KO neurons. These results are in accordance with existing literature indicating generally increased oxidative stress in the peripheral blood of PD patients (Chen et al., 2009). GSH is a vital cellular protective antioxidant, and the cellular ratio of GSH to GSSG is often used as a marker of cellular toxicity (Sentellas et al., 2014). The reduced glutathione redox ratio (GSH/GSSG) in PARK2 KO neurons is consistent with the concept of oxidative stress being a major component of the pathogenesis of nigral cell death in PD (Dias et al., 2013; Sentellas et al., 2014; Zitka et al., 2012). NAD ${ }^{+}$and NADH are fundamental molecules in metabolism and redox signaling (Chen et al., 2009). We found perturbed balance between $\mathrm{NAD}^{+}$and NADH molecules, pointing to a significantly lower level of $\mathrm{NAD}^{+}$ than NADH in the PARK2 KO neurons. The consequence of $\mathrm{NAD}^{+} / \mathrm{NADH}$ redox imbalance is initially reductive stress, which eventually leads to oxidative stress and oxidative damage to macromolecules such as DNA, lipids, and proteins (Yan, 2018). In line with our findings, loss of redox homeostasis has previously been associated with many pathological conditions, including PD (Wu et al., 2017).

In conclusion, in this study we sought to obtain a comprehensive description of the metabolomic changes in a PARK2 KO iPSC model of PD. Taken together, our results suggest that mitochondrial defects and subsequent changes in energy metabolism and oxidative stress combined with inefficient oxidative stress defense mechanisms may be associated with cellular dysregulation in PD and could represent a potential therapeutic target.

\section{EXPERIMENTAL PROCEDURES}

All detailed experimental procedures are available in the supplemental information. 
In vitro propagation and differentiation of neural stem cells

Isogenic PARK2 KO and healthy control neural stem cell (NSC) cell lines were provided by XCell Science Inc. (Novato, CA). NSCs were characterized and propagated according to standard protocols. Dopaminergic differentiation was achieved by culturing NSCs in DOPA Induction and Maturation Medium (XCell Science Inc., Novato, CA) according to the manufacturer's instructions for at least 25 days from NSC stage (39 days from iPSC stage) (Figures S5 and S6). See supplemental information for further details.

Mass spectrometry-based metabolomics

Sample collection and metabolite extraction were performed in a polar, ice-cold solvent (50\% methanol [Sigma], 30\% acetonitrile [Sigma], 20\% water [Merck Millipore]) as described in the supplemental information. The samples were analyzed by LC-MS analysis using an Agilent Zorbax Eclipse Plus C18 column and a Discovery HSF5 high-performance liquid chromatography column. The acquired raw data were converted to mzXML format and properly pre-processed prior to statistical analysis. Generated compound lists were uploaded to MetaboAnalyst.ca. See supplemental information for detailed procedures.

MMP, ATP, lactate, glucose uptake, $\mathrm{NAD}^{+} / \mathrm{NADH}$, ROS evaluation

MMP was investigated using the TMRE Mitochondrial Membrane Potential Assay Kit (Abcam). Intracellular ATP level at steady state was measured using ATPlite Assay Kit (PerkinElmer). Lactate level was measured using Lactate-Glo Assay Kit (Promega). Glucose uptake was assessed using Glucose Uptake-Glo Assay Kit (Promega). NAD ${ }^{+}$ and NADH levels were measured using NAD/NADH-Glo Assay Kit (Promega). ROS level was measured using ROS-Glo Assay Kit (Promega). The assays were performed according to the manufacturer's instructions. See supplemental information for detailed procedures.

\section{TEM}

Cells were seeded on 13-mm Thermanox plastic coverslips (Nunc) coated with poly-L-ornithin/laminin. Ultra-thin $(50-70 \mathrm{~nm}) \mathrm{sec}-$ tions were prepared, contrasted with $2 \%$ uranyl acetate (PolyScience) and lead citrate, and examined using a Philips CM100 transmission electron microscope equipped with a Morada camera. See supplemental information for further details.

\section{Statistical analysis}

Statistical analysis was performed using GraphPad Prism 7.0 software. To analyze the data, two-tailed unpaired Student's t test, multiple $t$ test, and one- or two-way ANOVA with multiple comparison test were applied. Data were considered statistically significant at $\mathrm{p}<$ $\left.0.05{ }^{(*)}, \mathrm{p}<0.01{ }^{(*}\right)$, and $\left.\mathrm{p}<0.001{ }^{(* *}\right)$. Data are presented as mean \pm standard error of the mean (SEM). Metabolomic fold change data were analyzed in MetaboAnalyst software using Student's t test.

\section{SUPPLEMENTAL INFORMATION}

Supplemental information can be found online at https://doi.org/ 10.1016/j.stemcr.2021.04.022.

\section{AUTHOR CONTRIBUTIONS}

J.O. and M.M. designed research. J.O., J.F.H., M.R., S.I.S., H.B., R.H.R., and B.J.R. performed experiments. N.J.F., P.H., S.A.C., and R.W.M. contributed new reagents or analytic tools. J.O., J.F.H., M.R., S.I.S., and H.B. analyzed data. J.O. and M.M. wrote the manuscript. J.F.H., M.R., S.I.S., H.B., N.J.F., and P.H. revised the manuscript for content. All authors read and approved the final manuscript.

\section{DECLARATION OF INTERESTS}

All authors declare that they have no competing interests.

\section{ACKNOWLEDGMENTS}

The authors thank Dorte Lyholmer, Nadine Becker-von Buch, Ulla Melchior Hansen, and Maria Pihl for excellent technical assistance, and Dr. Claire Gudex for editing the manuscript. The live imaging experiments were performed at DaMBIC, a bioimaging core facility at the University of Southern Denmark. DaMBIC was established by an equipment grant from the Danish Agency for Science, Technology and Innovation and by internal funding from the University of Southern Denmark.

The research leading to these results was supported by the Innovation Fund Denmark (BrainStem; 4108-00008A), H. Lundbeck A/ $S$, the Danish Parkinson Foundation, the Jascha Foundation, the A.P. Møller Foundation for the Advancement of Medical Science (15-396, 14-427), and the Faculty of Health Sciences at the University of Southern Denmark.

Reprogramming of the patient fibroblasts was conducted in the James Martin Stem Cell Facility, University of Oxford, and was supported by the Monument Trust Discovery Award from Parkinson's UK, The Oxford Martin School (LC0910-004), and the Innovative Medicines Initiative Joint Undertaking under grant agreement (115439), resources of which are composed of financial contribution from the European Union's Seventh Framework Program (FP7/2007e2013) and EFPIA companies' in kind contribution. We thank the High-Throughput Genomics Group at the Wellcome Trust Center for Human Genetics, Oxford (funded by Wellcome Trust grant 090532/Z/09/Z and MRC Hub grant G0900747 91070) for the generation of Illumina genotyping data.

Received: September 8, 2020

Revised: April 30, 2021

Accepted: April 30, 2021

Published: May 27, 2021

\section{REFERENCES}

Anandhan, A., Jacome, M.S., Lei, S., Hernandez-Franco, P., Pappa, A., Panayiotidis, M.I., Powers, R., and Franco, R. (2017). Metabolic dysfunction in Parkinson's disease: bioenergetics, redox homeostasis and central carbon metabolism. Brain Res. Bull. 133, 12-30.

Andersen, A.D., Binzer, M., Stenager, E., and Gramsbergen, J.B. (2017). Cerebrospinal fluid biomarkers for Parkinson's disease - a systematic review. Acta Neurol. Scand. 135, 34-56.

Andersen, L.W., Mackenhauer, J., Roberts, J.C., Berg, K.M., Cocchi, M.N., and Donnino, M.W. (2013). Etiology and therapeutic 
approach to elevated lactate levels. Mayo Clin. Proc. 88, 11271140 .

Berg, J.M., Tymoczko, J.L., and Stryer, L. (2002). The Glycolytic Pathway Is Tightly Controlled. Biochemistry, Fifth Edition (W.H. Freeman), Section 16.2.

Bertolin, G., Ferrando-Miguel, R., Jacoupy, M., Traver, S., Grenier, K., Greene, A.W., Dauphin, A., Waharte, F., Bayot, A., Salamero, J., et al. (2013). The TOMM machinery is a molecular switch in PINK1 and PARK2/PARKIN-dependent mitochondrial clearance. Autophagy 9, 1801-1817.

Bjorkblom, B., Maple-Grodem, J., Puno, M.R., Odell, M., Larsen, J.P., and Moller, S.G. (2014). Reactive oxygen species-mediated DJ-1 monomerization modulates intracellular trafficking involving karyopherin beta2. Mol. Cell Biol. 34, 3024-3040.

Blaszczyk, J.W. (2018). The emerging role of energy metabolism and neuroprotective strategies in Parkinson's disease. Front. Aging Neurosci. 10, 301.

Bogetofte, H., Jensen, P., Okarmus, J., Schmidt, S.I., Agger, M., Ryding, M., Norregaard, P., Fenger, C., Zeng, X., Graakjaer, J., et al. (2019a). Perturbations in RhoA signalling cause altered migration and impaired neuritogenesis in human iPSC-derived neural cells with PARK2 mutation. Neurobiol. Dis. 132, 104581.

Bogetofte, H., Jensen, P., Ryding, M., Schmidt, S.I., Okarmus, J., Ritter, L., Worm, C.S., Hohnholt, M.C., Azevedo, C., Roybon, L., et al. (2019b). PARK2 mutation causes metabolic disturbances and impaired survival of human iPSC-derived neurons. Front. Cell. Neurosci. 13, 297.

Bonifati, V., Rizzu, P., van Baren, M.J., Schaap, O., Breedveld, G.J., Krieger, E., Dekker, M.C., Squitieri, F., Ibanez, P., Joosse, M., et al. (2003). Mutations in the DJ-1 gene associated with autosomal recessive early-onset parkinsonism. Science 299, 256-259.

Bousquet, M., Calon, F., and Cicchetti, F. (2011). Impact of $\omega-3$ fatty acids in Parkinson's disease. Ageing Res. Rev. 10, 453-463.

Bratic, I., and Trifunovic, A. (2010). Mitochondrial energy metabolism and ageing. Biochim. Biophys. Acta 1797, 961-967.

Caviness, J.N. (2014). Pathophysiology of Parkinson's disease behavior-a view from the network. Parkinsonism Relat. Disord. 20, S39-S43.

Chan, D.C. (2020). Mitochondrial dynamics and its involvement in disease. Annu. Rev. Pathol. 15, 235-259.

Chen, C.M., Liu, J.L., Wu, Y., Chen, Y.C., Cheng, H.S., Cheng, M.L., and Chiu, D.T. (2009). Increased oxidative damage in peripheral blood correlates with severity of Parkinson's disease. Neurobiol. Dis. 33, 429-435.

Chinopoulos, C. (2013). Which way does the citric acid cycle turn during hypoxia? The critical role of alpha-ketoglutarate dehydrogenase complex. J. Neurosci. Res. 91, 1030-1043.

Chung, S.Y., Kishinevsky, S., Mazzulli, J.R., Graziotto, J., Mrejeru, A., Mosharov, E.V., Puspita, L., Valiulahi, P., Sulzer, D., Milner, T.A., et al. (2016). Parkin and PINK1 patient iPSC-derived midbrain dopamine neurons exhibit mitochondrial dysfunction and alphasynuclein accumulation. Stem Cell Reports 7, 664-677.

Crie, J.S., and Wildenthal, K. (1980). Influence of acidosis and lactate on protein degradation in adult and fetal hearts. J. Mol. Cell. Cardiol. 12, 1065-1074.
Delaney, N.F., Sharma, R., Tadvalkar, L., Clish, C.B., Haller, R.G., and Mootha, V.K. (2017). Metabolic profiles of exercise in patients with McArdle disease or mitochondrial myopathy. Proc. Natl. Acad. Sci. U S A 114, 8402-8407.

Dias, V., Junn, E., and Mouradian, M.M. (2013). The role of oxidative stress in Parkinson's disease. J. Parkinsons Dis. 3, 461-491.

Dimroth, P., Kaim, G., and Matthey, U. (2000). Crucial role of the membrane potential for ATP synthesis by F(1)F(o) ATP synthases. J. Exp. Biol. 203, 51-59.

Errea, O., Moreno, B., Gonzalez-Franquesa, A., Garcia-Roves, P.M., and Villoslada, P. (2015). The disruption of mitochondrial axonal transport is an early event in neuroinflammation. J. Neuroinflammation 12, 152.

Fernie, A.R., Carrari, F., and Sweetlove, L.J. (2004). Respiratory metabolism: glycolysis, the TCA cycle and mitochondrial electron transport. Curr. Opin. Plant Biol. 7, 254-261.

Fitzgerald, J.C., Camprubi, M.D., Dunn, L., Wu, H.C., Ip, N.Y., Kruger, R., Martins, L.M., Wood, N.W., and Plun-Favreau, H. (2012). Phosphorylation of HtrA2 by cyclin-dependent kinase-5 is important for mitochondrial function. Cell Death Differ. 19, 257-266.

Forcisi, S., Moritz, F., Lucio, M., Lehmann, R., Stefan, N., and Schmitt-Kopplin, P. (2015). Solutions for low and high accuracy mass spectrometric data matching: a data-driven annotation strategy in nontargeted metabolomics. Anal. Chem. 8, 8917-8924.

Gibson, G.E., Kingsbury, A.E., Xu, H., Lindsay, J.G., Daniel, S., Foster, O.J., Lees, A.J., and Blass, J.P. (2003). Deficits in a tricarboxylic acid cycle enzyme in brains from patients with Parkinson's disease. Neurochem. Int. 43, 129-135.

Grünewald, A., Voges, L., Rakovic, A., Kasten, M., Vandebona, H., Hemmelmann, C., Lohmann, K., Orolicki, S., Ramirez, A., Schapira, A.H., et al. (2010). Mutant Parkin impairs mitochondrial function and morphology in human fibroblasts. PLoS One 5, e12962.

Haenseler, W., Sansom, S.N., Buchrieser, J., Newey, S.E., Moore, C.S., Nicholls, F.J., Chintawar, S., Schnell, C., Antel, J.P., Allen, N.D., et al. (2017). A highly efficient human pluripotent stem cell microglia model displays a neuronal-Co-culture-specific expression profile and inflammatory response. Stem Cell Reports 8, 1727-1742.

Havelund, J.F., Andersen, A.D., Binzer, M., Blaabjerg, M., Heegaard, N.H.H., Stenager, E., Faergeman, N.J., and Gramsbergen, J.B. (2017). Changes in kynurenine pathway metabolism in Parkinson patients with L-DOPA-induced dyskinesia. J. Neurochem. 142, 756-766.

Haylett, W., Swart, C., van der Westhuizen, F., van Dyk, H., van der Merwe, L., van der Merwe, C., Loos, B., Carr, J., Kinnear, C., and Bardien, S. (2016). Altered mitochondrial respiration and other features of mitochondrial function in parkin-mutant fibroblasts from Parkinson's disease patients. Parkinsons Dis. 2016, 1819209.

Hou, Y., Dan, X., Babbar, M., Wei, Y., Hasselbalch, S.G., Croteau, D.L., and Bohr, V.A. (2019). Ageing as a risk factor for neurodegenerative disease. Nat. Rev. Neurol. 15, 565-581.

Imaizumi, Y., Okada, Y., Akamatsu, W., Koike, M., Kuzumaki, N., Hayakawa, H., Nihira, T., Kobayashi, T., Ohyama, M., Sato, S., et al. (2012). Mitochondrial dysfunction associated with increased 
oxidative stress and $\alpha$-synuclein accumulation in PARK2 iPSCderived neurons and postmortem brain tissue. Mol. Brain 5, 35.

Jourdain, P., Allaman, I., Rothenfusser, K., Fiumelli, H., Marquet, P., and Magistretti, P.J. (2016). L-Lactate protects neurons against excitotoxicity: implication of an ATP-mediated signaling cascade. Sci. Rep. 6, 21250.

Jurica, M.S., Mesecar, A., Heath, P.J., Shi, W., Nowak, T., and Stoddard, B.L. (1998). The allosteric regulation of pyruvate kinase by fructose-1,6-bisphosphate. Structure 6, 195-210.

Kim, K.Y., Stevens, M.V., Akter, M.H., Rusk, S.E., Huang, R.J., Cohen, A., Noguchi, A., Springer, D., Bocharov, A.V., Eggerman, T.L., et al. (2011). Parkin is a lipid-responsive regulator of fat uptake in mice and mutant human cells. J. Clin. Invest. 121, 3701-3712.

Lang, C., Campbell, K.R., Ryan, B.J., Carling, P., Attar, M., Vowles, J., Perestenko, O.V., Bowden, R., Baig, F., Kasten, M., et al. (2019). Single-cell sequencing of iPSC-dopamine neurons reconstructs disease progression and identifies HDAC4 as a regulator of Parkinson cell phenotypes. Cell Stem Cell 24, 93-106.e6.

Leite, T.C., Coelho, R.G., da Silva, D., Coelho, W.S., Marinho-Carvalho, M.M., and Sola-Penna, M. (2011). Lactate downregulates the glycolytic enzymes hexokinase and phosphofructokinase in diverse tissues from mice. FEBS Lett. 585, 92-98.

Lesage, S., and Brice, A. (2009). Parkinson's disease: from monogenic forms to genetic susceptibility factors. Hum. Mol. Genet. 18, R48-R59.

LeWitt, P. (2012). Recent advances in CSF biomarkers for Parkinson's disease. Parkinsonism Relat. Disord. 18, S49-S51.

Longo, N., Frigeni, M., and Pasquali, M. (2016). Carnitine transport and fatty acid oxidation. Biochim. Biophys. Acta 1863, 2422-2435.

Lucking, C.B., Durr, A., Bonifati, V., Vaughan, J., de Michele, G., Gasser, T., Harhangi, B.S., Meco, G., Denefle, P., Wood, N.W., et al. (2000). Association between early-onset Parkinson's disease and mutations in the parkin gene. N. Engl. J. Med. 342, 1560-1567.

Martinez, T.N., and Greenamyre, J.T. (2012). Toxin models of mitochondrial dysfunction in Parkinson's disease. Antioxid. Redox Signal. 16, 920-934.

Meredith, G.E., and Rademacher, D.J. (2011). MPTP mouse models of Parkinson's disease: an update. J. Parkinsons Dis. 1, 19-33.

Mihalik, S.J., Goodpaster, B.H., Kelley, D.E., Chace, D.H., Vockley, J., Toledo, F.G., and Delany, J.P. (2010). Increased levels of plasma acylcarnitines in obesity and type 2 diabetes and identification of a marker of glucolipotoxicity. Obesity (Silver Spring) 18, 1695-1700.

Nagesh Babu, G., Gupta, M., Paliwal, V.K., Singh, S., Chatterji, T., and Roy, R. (2018). Serum metabolomics study in a group of Parkinson's disease patients from northern India. Clin. Chim. Acta 480, 214-219.

Nita, M., and Grzybowski, A. (2016). The role of the reactive oxygen species and oxidative stress in the pathomechanism of the age-related ocular diseases and other pathologies of the anterior and posterior eye segments in adults. Oxid. Med. Cell. Longev. $2016,3164734$.
Nsiah-Sefaa, A., and McKenzie, M. (2016). Combined defects in oxidative phosphorylation and fatty acid beta-oxidation in mitochondrial disease. Biosci. Rep. 36, e00313.

Okarmus, J., Bogetofte, H., Schmidt, S.I., Ryding, M., García-López, S., Ryan, B.J., Martínez-Serrano, A., Hyttel, P., and Meyer, M. (2020). Lysosomal perturbations in human dopaminergic neurons derived from induced pluripotent stem cells with PARK2 mutation. Sci. Rep. 10, 10278.

Okuzumi, A., Hatano, T., Ueno, S.I., Ogawa, T., Saiki, S., Mori, A., Koinuma, T., Oji, Y., Ishikawa, K.I., Fujimaki, M., et al. (2019). Metabolomics-based identification of metabolic alterations in PARK2. Ann. Clin. Transl. Neurol. 6, 525-536.

Parker, W.D., Jr., Parks, J.K., and Swerdlow, R.H. (2008). Complex I deficiency in Parkinson's disease frontal cortex. Brain Res. 1189, 215-218.

Pickrell, A.M., and Youle, R.J. (2015). The roles of PINK1, parkin, and mitochondrial fidelity in Parkinson's disease. Neuron 85, 257-273.

Reed, X., Bandres-Ciga, S., Blauwendraat, C., and Cookson, M.R. (2019). The role of monogenic genes in idiopathic Parkinson's disease. Neurobiol. Dis. 124, 230-239.

Ren, Y., Jiang, H., Hu, Z., Fan, K., Wang, J., Janoschka, S., Wang, X., Ge, S., and Feng, J. (2015). Parkin mutations reduce the complexity of neuronal processes in iPSC-derived human neurons. Stem Cells 33, 68-78.

Ross, J.M., Oberg, J., Brene, S., Coppotelli, G., Terzioglu, M., Pernold, K., Goiny, M., Sitnikov, R., Kehr, J., Trifunovic, A., et al. (2010). High brain lactate is a hallmark of aging and caused by a shift in the lactate dehydrogenase A/B ratio. Proc. Natl. Acad. Sci. U S A 107, 20087-20092.

Saiki, S., Hatano, T., Fujimaki, M., Ishikawa, K.I., Mori, A., Oji, Y., Okuzumi, A., Fukuhara, T., Koinuma, T., Imamichi, Y., et al. (2017). Decreased long-chain acylcarnitines from insufficient beta-oxidation as potential early diagnostic markers for Parkinson's disease. Sci. Rep. 7, 7328.

Schapira, A.H. (2008). Mitochondria in the aetiology and pathogenesis of Parkinson's disease. Lancet Neurol. 7, 97-109.

Schapira, A.H. (2009). Etiology and pathogenesis of Parkinson disease. Neurol. Clin. 27, 583-603.

Sentellas, S., Morales-Ibanez, O., Zanuy, M., and Alberti, J.J. (2014). GSSG/GSH ratios in cryopreserved rat and human hepatocytes as a biomarker for drug induced oxidative stress. Toxicol. Vitro 28, 1006-1015.

Sharma, S., and Black, S.M. (2009). Carnitine homeostasis, mitochondrial function, and cardiovascular disease. Drug Discov. Today Dis. Mech. 6, e31-e39.

Stoessel, D., Schulte, C., Teixeira dos Santos, M.C., Scheller, D., Rebollo-Mesa, I., Deuschle, C., Walther, D., Schauer, N., Berg, D., Nogueira da Costa, A., et al. (2018). Promising metabolite profiles in the plasma and CSF of early clinical Parkinson's disease. Front. Aging Neurosci 10, 51.

Therond, P. (2006). [Oxidative stress and damages to biomolecules (lipids, proteins, DNA)]. Ann. Pharm. Fr. 64, 383-389.

Trezzi, J.P., Galozzi, S., Jaeger, C., Barkovits, K., Brockmann, K., Maetzler, W., Berg, D., Marcus, K., Betsou, F., Hiller, K., et al. 
(2017). Distinct metabolomic signature in cerebrospinal fluid in early Parkinson's disease. Mov. Disord. 32, 1401-1408.

Willkommen, D., Lucio, M., Moritz, F., Forcisi, S., Kanawati, B., Smirnov, K.S., Schroeter, M., Sigaroudi, A., Schmitt-Kopplin, P., and Michalke, B. (2018). Metabolomic investigations in cerebrospinal fluid of Parkinson's disease. PLoS One 13, e0208752.

Wu, J., Jin, Z., and Yan, L.J. (2017). Redox imbalance and mitochondrial abnormalities in the diabetic lung. Redox Biol. 11, 51-59.
Yan, L.J. (2018). Redox imbalance stress in diabetes mellitus: role of the polyol pathway. Anim. Model Exp. Med. 1, 7-13.

Zitka, O., Skalickova, S., Gumulec, J., Masarik, M., Adam, V., Hubalek, J., Trnkova, L., Kruseova, J., Eckschlager, T., and Kizek, R. (2012). Redox status expressed as GSH:GSSG ratio as a marker for oxidative stress in paediatric tumour patients. Oncol. Lett. 4, 1247-1253.

Zorov, D.B., Juhaszova, M., and Sollott, S.J. (2014). Mitochondrial reactive oxygen species (ROS) and ROS-induced ROS release. Physiol. Rev. 94, 909-950. 